\title{
Neue Weiterbildung Intensivmedizin: Bewährtes Konzept mit neuen kompetenzbasierten Inhalten
}

\author{
G. Marx, H. Van Aken
}

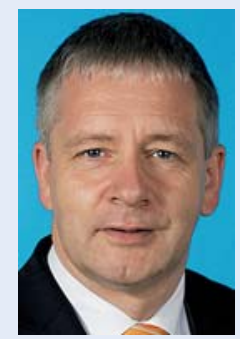

Gernot Marx



Hugo Van Aken
Korrespondenzadresse Univ.-Prof. Dr. med. Gernot Marx, FRCA Direktor der Klinik für Operative Intensivmedizin und Intermediate Care Universitätsklinikum der RWTH Aachen

Pauwelsstr. 30 52074 Aachen E-Mail: gmarx@ukaachen. de

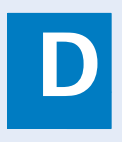

ie Intensivmedizin ist ein sehr komplexer Bereich in der modernen Medizin, welcher ohne jeden Zweifel eine spezielle Weiterbildung auf der Basis der verschiedenen Facharztqualifikationen erfordert. Kritisch kranke Patienten benötigen nicht nur eine kontinuierliche 24-stündige Betreuung durch einen Arzt, sondern durch einen Arzt, der den besonderen Herausforderungen gewachsen ist. Deswegen wurde in den 2013 publizierten überarbeiteten Qualitätsindikatoren Intensivmedizin aufgenommen, dass zumindest in der Kernarbeitszeit ein Facharzt mit Zusatzqualifikation Intensivmedizin auf der Intensivstation präsent sein muss [1]. Die Grundlagen der ärztlichen Weiterbildung werden auf europäischer Ebene in der UEMS (Union Européenne des Médicins Spécialistes - Vereinigung der Europäischen Medizinischen Fachgesellschaften) geregelt, in der alle medizinischen Fachdisziplinen repräsentiert sind. Für die Intensivmedizin als interdisziplinäres Fachgebiet wurde 1999 im Rahmen der UEMS ein interdisziplinäres Komitee gegründet, das „Multidisciplinary Joint Committee of Intensive Care Medicine (MJCICM)“, welches sich seitdem mit den speziellen Anforderungen in dem komplexen Bereich beschäftigt $[2,3]$.

Die operative Intensivmedizin in Deutschland wird sehr häufig von Anästhesiologen betrieben. Dies ist eine konsequente Entwicklung, da die perioperative Behandlung von Vitalfunktionen und die kooperativinterdisziplinäre Ausrichtung die Anästhesiologie dazu prädestiniert [2,3]. Andererseits profitieren z.B. internistisch erkrankte Patienten sehr von der Kompetenz eines kardiologisch oder pulmonologisch ausgebildeten Intensivmediziners. In Deutschland wird zurzeit eine neue Musterweiterbildungsordnung (MWBO) erarbeitet. Die Inhalte dieser neuen MWBO sind kompetenzbasiert und können deswegen sehr differenziert abgebildet werden. Die zentrale Komponente der neu- en MWBO ist nicht die Zahl der Inhalte, sondern vor allem die Kompetenz, die erworben werden muss. In der Entwicklung der neuen MWBO für die Zusatzqualifikation Intensivmedizin wurde gemeinsam durch die in der DIVI (Deutsche interdisziplinäre Vereinigung für Intensiv- und Notfallmedizin) organisierten verschiedenen Fachbereiche (Anästhesiologie, Chirurgie, Innere Medizin, Neurologie und Neurochirurgie sowie Pädiatrie) die Fortführung des bewährten kombinierten Weiterbildungskonzepts in Deutschland bestätigt und betont: die interdisziplinäre Weiterbildung eines Intensivmediziners einerseits, in Kombination mit fachspezifischer Expertise andererseits, die auf der jeweiligen Facharztausbildung basiert. Dieses bewährte Konzept wird in der neuen MWBO durch kompetenzbasierte Weiterbildungsinhalte weiterentwickelt und verbessert.

So kann auch zukünftig gewährleistet werden, dass kritisch kranke Patienten optimal versorgt werden und die jeweils individuell benötigte Diagnostik und Therapie erhalten.

\section{Literatur}

1 Braun JP et al. Intensivmedizinische Qualitätsindikatoren für Deutschland 2013 - zweite Auflage. GMS German Medical Science 2013; 11, ISSN1612-3174

2 De Lange S, Van Aken H, Burchardi H. European Society of Intensive Care Medicine statement: intensive care medicine in Europe-structure, organisation and training guidelines of the Multidisciplinary Joint Committee of Intensive Care Medicine (MJCICM) of the European Union of Medical Specialists (UEMS). Intensive Care Med 2002; 28: 1505-1511

3 Marx G, Leuwer M. Intensivmedizin morgen. Anästhesiol Intensmed Notfallmed Schmerzther 2003; 38: 273-276 